\title{
Ethics and Law in Congenital Defects
}

José M Carrera

President of Matres Mundi and Secretary General of International Association of Perinatal Medicine, Barcelona, Spain

Correspondence: José M Carrera, President of Matres Mundi and Secretary General of International Association of Perinatal Medicine, C/Londres, 6-puerta 8, 08029-Barcelona, Spain, e-mail: jmcarrera@matres-mundi.org

\section{INTRODUCTION}

Prenatal diagnosis of congenital defects includes all medical procedures that lead to the detection or diagnosis of any fetal anomaly.

It is a relatively new medical speciality, with no more than 40 years in existence, and it requires complex and expensive technology, as well as extensive training.

Many ethical, social and legal conflicts have arisen in this area of medicine, for several reasons, amongst these: The complexity of the issue at stake (there are thousands of different congenital defects), the degree of sophistication of the technology required (implying substantial investment from hospitals), the need for experts in prenatal diagnosis (not all gynecologists are qualified), the exclusion experienced by individuals with suffering from a deficiency, and, above all, the ideological background that underlies the decision making process.

In the last few years, there has been as much development in the domain of bioethics, as in the choices that are taken after the detection and/or diagnosis of fetal congenital defects. With regards to bioethics, this incorporates legislation, regulations, codes of conduct and laws that serve to direct the medical advice and the use of the technology used when making a prenatal diagnosis (ultrasound, invasive examination, etc). There is no doubt that this is a delicate area, considering, the particular vulnerability of the fetus and the difficulty in clearly establishing its rights.

The discipline of bioethics is a scientific attempt to analyze the problems that often arise in the health field, due to difference of opinion, and at times, conflicting personal and social values. In this case, it is an analysis of the issues found within domain of prenatal diagnosis. The disciplines's fundamental methodology is composed and rational reflection, as well as open dialogue and multidisciplinary approaches. ${ }^{1,2}$

Legal requirements are the result of society attempting to protect the rights of individuals using laws, as well as trying to determine their duties when faced with specific situations. Theoretically, the objective of legal requirements is to prioritise and guarantee mutual well-being ahead of private interests, but in practice, these requirements are influenced by prevailing ideology (e.g. political, religious, social elements, and the culture and socioeconomic development level of a country).

In democratic countries, the lack of uniformity with regards to ethical values is often manifested through permissive legislation, which tolerates a pluralism of conducts as long as inalienable rights of third parties are not violated. ${ }^{3}$

\section{GENERAL ETHICAL PRINCIPLES}

The term "general ethical principles" refers to the general criteria that serve as a basis for writing and justifying the majority of "good practice" regulations that govern Medicine, and which should also be the reference point for legislators. Naturally, these principles must adapt themselves to the diverse cultural traditions of each country. ${ }^{4,5}$ They can be summarized under three main headlines:

1. Principle of beneficence: This can be defined as the obligation to do our utmost to protect the welfare of the patient (in this case the fetus), maximising the benefits of our decisions and reducing the chances of harm or injury. It is the principle of our Latin master "primum nonnocere" ("first, do no harm").

2. Principle of autonomy: This principle obligates the doctor to respect the integrity of the values and beliefs of his/her patient, their patients' perspective with regards to their own interest, and to implement only the clinical strategies that they authorise. In practice, to comply with the requisites of this principle, an "informed consent" that clearly specifies the type of examination and the possible risks or injury that may occur must be read and signed by the patient. When the patient has diminished legal capacity (children, individuals with mental disabilities, etc) or is legally invalid (the fetus), this principle must ensure that these values are not breached.

In the case of the fetus, this principle only comes into consideration when the fetus can be considered "as a patient", with a considerable consensus, when the fetus is "viable". ${ }^{6-8}$ But naturally, "viability" is also an indefinable concept, as it depends on the medical infrastructure and technology, which differ across countries. 
3. Principle of justice: The introduction of this principle into Medicine has been made on the basis of "equity": every person has the right to receive the best treatment possible, in accordance with their special and specific needs.

\section{BASIC LEGAL PRINCIPLES}

Laws vary in different countries. For this reason, it is important not to confuse ethical principles with legal requirements. In many countries, not all legal requirements are ethical, and in the same way not all ethical principles are necessarily enforced by law.

In general, the legislation with regard to prenatal diagnosis and possible intrauterine therapies is common to many countries in that:

1. The physician that carries out the prenatal diagnosis technique or treatment must hold the appropriate qualifications, in other words, "an academic title".

2. The specialists (obstetricians, sonologists, radiologists, etc.) must have also undergone sufficient training in order to carry out such techniques, which involve a degree of risk to both the mother and to the fetus, lying within the normal range of risk as reported statistically.

3. Consent must be obtained from the patient. It must be observed that the notion of "informed consent" should not be regarded as solely an ethical requirement, but also as a legal requisite.

With regards to the legal induced termination of an established pregnancy (abortion), the majority of legislation states:

1. That it may only be carried out under certain circumstances (following rape, congenital defect present in the fetus, serious illness of the mother), and with provisional time limits determined dependent on each circumstance. In some countries abortion is an elective procedure, carried out under the pregnant women's wishes, up until a specified gestation period (generally 12-14 weeks).

2. That the interruption of the pregnancy should only be performed under adequate medical conditions, and accompanied by appropriate documentary and legal authorization.

\section{SPECIFIC LEGAL-ETHICAL PRINCIPLES IN PRENATAL DIAGNOSIS}

In prenatal diagnosis, some specific ethical principles must be adopted. ${ }^{9}$ The vast amount of these are usually also regulated by the majority of national legislation:

1. All pregnant women (and their partners) are entitled to have access to objective information about "congenital defects" (occurrence, prenatal diagnostic possibilities, specific risks, alternative options, etc).
2. Guided counseling is not ethical. The physician should not attempt to impose his/her personal view over patients.

3. All examinations should be carried out according to "lex artis" (sufficient expertise, appropriate technology and in a suitable environment).

4. The examination results must be confidential and access to such results should be restricted.

5. Information provided regarding the results should be adequate, and communicated in a way that can be easily understood by the couple.

6. Information regarding possibilities and options should be provided objectively, to help the couple reach a personal decision, and in accordance with their needs and beliefs.

\section{CHRONOLOGY OF ETHICAL AND LEGAL DILEMMAS}

Ethical and legal dilemmas normally arise at five different stages, which are linked to prenatal diagnosis. ${ }^{9}$

1. When disclosing the information.

2. When establishing the indication.

3. When performing the technique.

4. When communicating the diagnosis, and finally.

5. When making any subsequent decision.

In this chapter we will describe ethical aspects, especially those that are habitually reinforced by legal requirements, and we will focus our attention on the approved methods in the practice, as well as those that are considered inadequate and unacceptable.

1. Disclosing the information: At the moment that information is relayed to parents, it is necessary to remember that:

1.1. The information given should be adapted to the specifics of the couple: age, personal and family history, etc.

1.2. Information provided should include advice about the various detection methods available (biochemical and ultrasound screening) and for diagnosis (invasive testing, etc.)

1.3. With respect to the ultrasound examination, it should be explained that the medical evidence shows that all pregnant women should be examined at the proper time, using this procedure, since the majority of malformed fetuses are found in low-risk pregnancies.

1.4. The information content should be clear, comprehensive, continuous, understandable and consistent with the patient's culture and idiosyncrasy.

1.5. If the couple refuse the ultrasound, or any other prenatal diagnosis method, they must sign a statement of "nonconsent”.

It is very important to avoid inappropriate conduct: failing to inform the patient about congenital defects (ideological or religious reasons, professional negligence, etc.), providing biased information (under-estimating congenital defect risks or 
over-estimating the risks linked to the tests), and providing partial or incomplete information on the present possibilities of each test: what it does/does not do.

2. Establishing the indication: The norms accepted at the time of establishing the indication are:

2.1. The number and chronology of the ultrasound examinations to be performed are determined according to the history, maternal-fetal pathology and specific protocols.

2.2. The option of a particular test (for "detection" and/ or "diagnosis" purposes) basically depends on the indication, age, risk, and on the medical history of the pregnant woman (and her partner).

2.3. The choice for the invasive method (amniocentesis, chorionic biopsy or cordocentesis, etc.) is made on the basis of the indication, week of pregnancy and the experience of the physician.

2.4. A policy of excessive indications should be avoided. 2.5. Informed consent must be obtained, as mentioned.

At this moment it is necessary to avoid the following inappropriate actions: Selecting a method which is not suitable, due to personal limitations (lack of experience or poor resources), selecting an invasive test without any specific indications, and failing to recommend invasive tests in cases where they should be carried out (high-risk for biochemical or ultrasound screening, etc).

3. Performing the technique: This stage is of course, very important. I believe that it is necessary to observe the following to comply with 4 basic rules:

3.1. The operator should be sufficiently qualified and experienced. The technique should be performed with an appropriate level of care.

3.2. The technique must be carried out in an appropriate environment (with privacy, to ensure peace of mind, reduced family pressure and suitable technological structure, etc).

3.3. The technique must be carried out following specific protocol and with safety standards that must be observed in all cases.

3.4. If the physician does not have the required experience, and/or the appropriate technology, the patient should be referred to another prenatal center with the necessary and confirmed experience.

Bad practice is evident in the following situations: carrying out the technique without the necessary experience (increasing the level of risk), carrying out invasive testing in a negligent manner (incomplete asepsis, etc.), failing to take the steps required to ensure the safety and confidentiality of the testing and of the ensuing results (promiscuity, verbal information given in public, mistakes, etc.), and not clearly informing prior to carrying out testing that performing a subsequent legal abortion will not be possible due to ideological reasons (i.e., due to religious beliefs, at confessional hospitals, practitioners with conscientious objection, etc).

4. Communicating the diagnosis: The communication of diagnosis to the couple is a very delicate and sensitive moment. Usually the accepted guidelines are:

4.1. The diagnosis must be communicated verbally to the patient, in person, by a member of medical staff.

4.2. In addition, the diagnosis (normal or pathological), must be delivered in writing, through an appropriate document.

4.3. It is desirable that the person responsible for informing the patient of the diagnosis is also the obstetrician in charge of the case.

4.4. The information should be communicated in a way that can be easily understood by the couple, particularly in the case of a pathological diagnosis.

4.5. In some cases, it is recommended that a "second opinion” be advised, from a list of specialized physicians or centers.

4.6. When necessary, special psychological and social support should be provided.

Regrettably, it is very common in some hospitals to observe mistakes of this kind: Untimely communication of the diagnosis by unqualified staff (administrative staff, etc.), communicating the information in an inadequate way (telephone or fax, via third persons, etc.), communicating the diagnosis with too much haste, or without previously analysing the case, etc.

5. Decision making following diagnosis: With regards to the moment following diagnosis, when it is necessary to make decisions, there are 7 accepted norms:

5.1. Before issuing any prognosis, and suggesting alternative options, a consistent diagnosis must be reached.

5.2. Sonologists should not rest satisfied with merely having detected a malformation. It is necessary to establish possible syndrome diagnosis (look for other anomalies) and carry out complementary testing (cytogenetic or biochemical studies).

5.3. No decision should be made without first having clearly defined the disorders of the fetus. If the fetal malformation is compatible with life, parents will be advised to speak to a pediatrician (Prenatal pediatric consultation). 
5.4. The "Hospital congenital defects committee" must examine the results of every test to evaluate the risks and advantages of any decision and option.

5.5. The information given to the couple, about different possibilities and options, must be easy to understand, clear and complete, and also suited to their personal circumstances.

5.6. The couple should be reminded of the need to carry out a necropsy study in the case of fetal death or legal abortion.

5.7. The physician must fully respect the decisions made by the patient and the couple, within the limits of the law.

The following must be considered as inappropriate practice: unduly delaying the decision as to whether terminate pregnancy (due to negligence or ideological convictions) until the legal time limit for abortion has been reached, giving onesided advice that presents one single option (legal abortion), without having discussed any other alternatives with the couple, causing the patient to opt for the termination of pregnancy on the basis of mere conjecture or for trivial reasons, failing to offer legal abortion in cases of severe fetal pathology, while underestimating the problems caused to the child in the future, and providing inaccurate or negligent information regarding the outcome and prospects of the necropsy study.

\section{OBSTETRICAL CONDUCT FOLLOWING PRENATAL DIAGNOSIS OF A CONGENITAL DEFECT}

Following the prenatal diagnosis of a congenital defect obstetrical diagnosis cannot be univocal. ${ }^{10-12}$ Irrespective of the legal and ethical considerations that we have put forward, as well as religious and social perspectives, which necessarily will vary from one geographical field to another, the obstetrician will have to consider the following questions before formulating possible alternatives:

1. The degree of reliability of the diagnosis: Have all the necessary and possible examinations been carried out? Are there other associated anomalies? Is there a chromosomal anomaly?

2. The gravity of the discovered defect: Is it compatible with life? To what extent may it affect the future quality of life?

3. Stage of pregnancy: Before or after the 22nd week? Is the fetus viable or not?

4. Possibilities for treatment: Can neonatal surgery be carried out? Is it possible to take a palliative measure or can it be resolved using prenatal methods? Does a medical treatment or a prenatal surgery exist?

5. Parents' opinion once they have been correctly informed: In this section, we will look at the possible responses according to the type of the congenital defect found present.
Response according to the type of defect:

There is notable accord for considering the legal induced termination of an established pregnancy (abortion), under the following circumstances:

1. Anatomical malformations that are not compatible with life; as is the case with some structural encephalic defects (anencephaly, porencephaly, hydranencephaly, etc).

2. Anatomical malformations that significantly compromise quality of life: Spina bifida, meningocele, hycrocephaly, agenesis of the corpus callosum, encephalocele, etc.

3. Serious congenital defects, associated with chromosome anomalies (aneuploidies, etc).

Specific legislation provide that the legal interruption of a pregnancy with the aforementioned indications should only be carried out before the 22nd week of pregnancy. Others, on the other hand are more compliant, or on the contrary, more restrictive.

There are certain defects sharing in common the fact that they become more severe as pregnancy progresses. Therefore, the possibility of a substantial improvement of the perinatal results exists, if a timely and selective fetal extraction is carried out, be it through induction (provocation) of labor or by cesarean section.

The following pathologies fall under this example: progressive obstructive hydronephrosis, progressive obstructive hydrocephaly, intestinal ischemia or necrosis secondary to meconium ileus or volvulus, and fetal hydropesia (immune or not).

In all cases the fetus should be mature or able to mature pharmacologically, and the chance that the defect can be corrected using neonatal methods must be present.

Certain fetuses with congenital defects should not be delivered or removed through the vagina, due to the risk of dystocia or possible injury to one of his/her organs. In these cases, an elective cesarean should be carried out. This applies to: hydrocephaly, (with macrocephaly), large teratoma sacrocoxigeo, large cystic hygroma or double monsters, and also in the case of meningoceles or large or ruptured omphaloceles.

At present there are a number of defects that can be treated "in utero", be it pharmalogically or surgically. ${ }^{13}$ Amongst these, metabolic disorders and cardiac rhythm disorders (cardio version) must be mentioned.

The most practiced surgical procedures are those that consist in fetal percutaneous incisions, guided by ultrasound, such as amnioreduction, septostomia, or the puncturing of cavities (thoracentesis, paracentesis, cystocentesis and derivatives, etc). Alongside these, fetal transfusion, through 
piercing the umbilical cord must be included (isoimmunization $\mathrm{Rh}$, etc.). An increasingly growing technique is fetal therapy through percutaneous laparoscopy (foetoscopy), in order to remedy feto-fetal transfusion syndrome in monochorial twins (occlusion by laser of the placental vascular anastomosis), amniotic constriction band suppression, and occlusion of the umbilical cord in the TRAP sequence (Twin Reversed Arterial Perfusion), etc.

On the other hand, exutero surgery (laparotomy and hysterotomy) runs the risk of significant problems of technique, that at present are limited to the field of investigation.

\section{CONCLUSION}

Prenatal diagnosis of congenital defects should be carried out under strict ethical criteria that respect the basic rights of mother and baby. ${ }^{14}$ However, all prenatal intervention regarding the fetus should take into consideration the condition of the "patient" and the condition that the fetus reaches when viability increases.

The choice to interrupt pregnancy (abortion) due to the presence of a congenital defect must adhere to the law, the principle of autonomy of each patient and the possible conscientious objection of practitioners. In all cases, the obstetrician must make all efforts to respect and protect the dignity of human life.

\section{REFERENCES}

1. Stirrat GM. Education in perinatal ethics. In Kurjak A and Chervenak FA. Textbook of Perinatal Medicine (2nd ed) London: Informa 2006;146-57.

2. Beauchamp TL, Childress JF. Principles of biomedical ethics (3rd ed.) New York: Oxford University Press 1989.
3. Carrera JM. Aspectos éticosy legales de la investigación clínica en Medicina Perinatal. In Fabre E, et al. Investigación Clínica en Perinatalogia. Barcelona: Masson 1998;477-90.

4. The National Commission for the protection of Human Subjects of Biomedical and Behavioral Research. The Belmont Report. Dhew Publication 1978;5:78-112.

5. Chervenak FA Mc, Cullough LB. Ethics: An essential dimension of perinatal Medicine. In “Textbook of Perinatal Medicine” (2nd ed.) Edit by A Kurjak and FA Chervenak. Informa, London, 2006:129-38.

6. Chervenak FA Mc, Cullough LB. The fetus as a patient: Implications for diorective versus nondirective counselling for fetal benefit. Fetal Diagn Ther 1991;6:93-100.

7. Chervenak FA Mc, Cullough LB. Consideraciones éticas en medicina embrionaria. En: Medicina del Embrión Edit by: JM Carrera and A Kurjak. Masson. Barcelona 1997;661-620.

8. Chervenak FA Mc, Cullough LB. Perinatal ethics: A practival method of analysis of obligations to mother and fetus. Obstet Gynecol 1985;66:442-46.

9. Carrera JM. Boethical aspects of ultrasonographioc and invasive diagnosos. In: Contraversies in Perinatal Medicine Edit by: JM Carrera, FA Chervenak, A Kurjak. Partenon Publishing. London 2003;282-88.

10. Carrera JM, Mallafre J. Conducta obstétrica ante el diagnóstico prenatal de un defecto congénito. En: “Prenatal Diagnosis” Edit by JM Carrera. Salvat, Barcelona 1987;709-18.

11. Mc Cullogh LB, Chervenak FA. Ethics in Obstetrics and Gynecology. New York: Oxford University Press 1994.

12. Chervenack FA, Mc Cullough LB. What is obstetric ethics?. Perinat Med 1995;23:331-41.

13. Harrison MR, Filly RA, Prer JRT, et al: Management of the fetus with a correctable congenital defect. JAMA 1981;246:63539.

14. Schenker JG: Medical and ethical aspects of pre-embryo research. In: The fetus as a patient: The evolving challenge, Edit by: F Chervvenak, A Kurjak, Z Papp. Parthenon publishing, London 2002. 